\title{
"Integrated knowledge translation" for globally oriented public health practitioners and scientists: Framing together a sustainable transfrontier knowledge translation vision
}

This article was published in the following Dove Press journal:

Journal of Multidisciplinary Healthcare

29 May 2010

Number of times this article has been viewed

\section{Véronique Lapaige}

Faculty of Nursing, CIFSS, Laval University, Quebec City, QC, Canada

Correspondence:Véronique Lapaige Faculty of Nursing (Community Health), CIFSS (Centre intégré de formation en sciences de la santé), Room 3572, Laval University, $1050 \mathrm{Av}$ de la Médecine, Quebec City, QC, GIV 0A6, Canada Tel + I 4186562 I3I \#|255I, $+15143669854$

$\mathrm{Fax}+\mathrm{I} 4186567825$

Email veronique.lapaige@fsi.ulaval.ca
Abstract: The development of a dynamic leadership coalition between practitioners and researchers/scientists - which is known in Canada as integrated knowledge translation (KT) - can play a major role in bridging the know-do gap in the health care and public health sectors. In public health, and especially in globally oriented public health, integrated KT is a dynamic, interactive (collaborative), and nonlinear phenomenon that goes beyond a reductionist vision of knowledge translation, to attain inter-, multi-, and even transdisciplinary status. Intimately embedded in its socioenvironmental context and closely connected with the complex interventions of multiple actors, the nonlinear process of integrated $\mathrm{KT}$ is based on a double principle: (1) the principle of transcendence of frontiers (sectorial, disciplinary, geographic, cultural, and cognitive), and (2) the principle of integration of knowledge beyond these frontiers. However, even though many authors agree on the overriding importance of integrated KT, there is as yet little understanding of the causal framework of integrated KT. Here, one can ask two general questions. Firstly, what "determines" integrated KT? Secondly, even if one wanted to apply a "transfrontier knowledge translation" vision, how should one go about doing so? For example, what would be the nature and qualities of a representative research program that applied a "transfrontier collaboration" approach? This paper focuses on the determinants of integrated KT within the burgeoning field of knowledge translation research (KT research). The paper is based on the results of a concurrent mixed method design which dealt with the complexity of building and sustaining effective coalitions and partnerships in the health care and public health sectors. The aims of this paper are: (1) to present an "integrated KT" conceptual framework which is global-context-sensitive, and (2) to promote the incorporation of a new "transfrontier knowledge translation" approach/vision designed primary for globally oriented public health researchers and health scientists.

Keywords: determinants, conceptual framework, systematic review, mixed methods research, collaboration, partnership, knowledge-sharing, globalization, sustainability

\section{Introduction}

In today's globalized learning economy, ${ }^{1,2}$ the ability to integrate, share, and disseminate knowledge across functional silos in health care organizations constitutes a major challenge, referred to as a knowledge-sharing (KS) challenge in the knowledge management parlance ${ }^{3-8}$ or as a knowledge translation (KT) challenge by public health 
practitioners and scientists. ${ }^{9-14}$ In the context of contemporary globalization, the difficulty of this dual KS-KT health-related challenge is augmented by the need for social sustainability and eco-socio-efficiency. ${ }^{15-17}$

Improvements in the intertwined $\mathrm{KS}-\mathrm{KT}$ aspect of the public health practice and health care are based on the ability to generate and integrate new knowledge into clinical/managerial practice while maintaining sound clinical/managerial processes. However, the acquisition and integration of knowledge is mostly "bit by bit", fragmented, and unbalanced. Subsequently, and along with many authors, ${ }^{18-25} \mathrm{I}$ believe that an active collaboration or coalition for sustainable $\mathrm{KS}-\mathrm{KT}$ practices is the cornerstone to establishing effective knowledge-based health care institutions. ${ }^{16}$ The development of a dynamic leadership coalition between practitioners and researchers/ scientists - which is known in Canada as integrated KT - can play a major role in bridging the know-do gap in the health care and public health sectors. The term "integrated KT" refers to both a process and its result. In public health, and especially in globally oriented public health, integrated KT is a dynamic (nonstatic), interactive (collaborative), and nonlinear phenomenon that goes beyond a reductionist vision of knowledge translation (sometimes seen in clinical epidemiology), to attain inter-, multi-, and even transdisciplinary status. The process of integrated KT is based upon: (i) the collaboration of individual and institutional actors and the integration of their respective knowledge bases, (ii) the development of a sustained synergy among knowledge users and knowledge producers, and (iii) the emancipation of postpositivist biomedical paradigms, removal of interdisciplinary barriers, and the development of sectors favoring a collective approach to shared problems and questions concerning health.

Intimately embedded in its socioenvironmental context and closely connected with the complex actions and interventions of multiple actors, the nonlinear process of integrated KT is based on a double principle: (1) the principle of transcendence of frontiers (sectorial, disciplinary, geographic, cultural, and cognitive), and (2) the principle of integration of knowledge beyond these frontiers. Integrated KT thus becomes, according to Lapaige et al a double-process of transfrontier integration that permits: (i) optimization of evidencebased practice, evidence-based health care management, and evidence-informed policy, (ii) the development of ecolearning health care institutions, and (iii) the promotion of evidence-based, equitable, and sustainable public health in response to collective and individual vulnerabilities in a context of globalization. ${ }^{26}$ However, even though many authors ${ }^{18-26}$ agree on the overriding importance of integrated KT - the development of sustained partnerships between producers and consumers of knowledge - there is as yet little understanding of the causal framework of integrated KT. Here, one can ask two general questions. Firstly, what "determines" integrated KT? In other words, what are the success factors of coalitions and partnerships in KS and KT that actually (i) improved patient care, and (ii) developed ecolearning health care institutions? Secondly, even if one wanted to apply a "transfrontier knowledge translation" vision, how should one go about doing so? For example, what would be the nature and qualities of a representative research program that applied a "transfrontier collaboration" approach?

This paper focuses on the determinants of integrated KT within the burgeoning field of knowledge translation research. The paper is based on the results of a concurrent mixed method design which dealt with the complexity of building and sustaining effective coalitions and partnerships in the health care and public health sectors. The aims of this paper are: (1) to present an "integrated KT" conceptual framework which is global-context-sensitive, and subsequently (2) to promote the incorporation of a new "transfrontier knowledge translation" approach in health care and (global) public health research.

The paper is divided into four parts: (1) terminological clarifications, and particularly the similarities and differences between KT, KT research, and integrated KT, (2) presentation of the QUAN-qual mixed model design used in my KT research study; based on preliminary results of this study, (3) a conceptual framework of integrated KT, which highlights the growing need for a global-context-sensitive vision in KT and evidence-based decision-making in health care institutions; and (4) in conclusion, presentation of an example of a currently active Canadian research program which has embraced an innovative vision in KT research. This new globally oriented KT approach transcends six types of borders in bridging the know-do gap in health care and public health: it has been called "transfrontier KT approach".

\section{Knowledge translation, knowledge translation research, and integrated knowledge translation: Terminologies, similarities, and differences}

In today's information age, the importance of health-related knowledge as a key source of competitive advantage is 
well established worldwide, as suggested by the increasing literature focusing on health care knowledge creation (generation, production), (re)use, application, exchange, dissemination, and transfer, and the rapid growth of the KT research field in Canada. ${ }^{1,6-8,10-15,17,27-50}$

Over recent years, there has been a major shift in the world economy from an industrial economy to a knowledge economy. ${ }^{2,51,52}$ Canada, like other Western societies, has found itself in a context characterized by increasing globalization of production and exchange of goods, services, and capital, as this "new economy" spreads. The importance of knowledge as a source of sustainable competitive advantage has been discussed by a myriad of authors, and managing knowledge has become a major concern for many organizations, including health care institutions. ${ }^{4,5}$

At the dawn of the 21st Century, knowledge is a crucial resource in the provision of health care, guiding improvements to clinical/managerial decision-making, patient care, health outcomes, workforce quality, and organizational behavior and structure.

In this paper, evidence-based decision-making is discussed not only at the micro level of individuals (clinicians, public health practitioners), but also at the meso level of groups (teamwork) and sectors (health sector, public health field). According to Shortell et al the notions of KT (individual level) and KS (meso level) have been intertwined. ${ }^{46}$ This paper considers sustainable KS-KT practices - artifactmediated, experience-mediated, and resource-mediated KS-KT practices (Table 1) - and the determining forces of their success in order to (i) develop ecolearning health care institutions, and (ii) improve patient care, population health, and the working lives of both (public) health practitioners and scientists at a time of changing roles and expectations.

While there is an overlap between these two notions, KT and KS are conceptually distinct (Table 1). They are nevertheless complementary. KT is emerging worldwide as a paradigm to learn and act toward closing the know-do gap in the domain of health care and the field of public health. In this paper, I am focusing on integrated knowledge translation with public health practitioners and health care researchers (Table 1) at the micro (clinical level) and meso (managerial/organizational level) levels. The term "integrated KT" describes a different way of doing research in which researchers and research users work together to shape the research process, starting with collaboration on setting the research questions, deciding the methodology, being involved in data collection and methodological development, interpreting the findings, and helping to disseminate the research results (Table 2) ${ }^{53}$ This approach, described in terms such as practice-oriented translational research (T2 research), collaborative research, action-oriented research, and co-production of knowledge, should produce findings which are more likely be relevant to, and for, end users. ${ }^{54}$ Furthermore, in this paper, I consider KS, which is essentially used in the knowledge management parlance ${ }^{3,4}$ and better tailored for a meso level, as a part of integrated KT (Figure 1).

In this paper, the basic premise is that $\mathrm{KT}$ is a multiactor and translevel phenomenon involving different levels of evidence-based decision-making and, correspondingly, different types of knowledge (Figure 1). Health care knowledge is a social construct and the processes of $\mathrm{KS}$, $\mathrm{KT}$, and integrated KT (acquiring knowledge; [re]using knowledge; developing new knowledge) are social processes and entail organizational changes. Linking integrated KT with organizational learning can usefully interconnect different levels within a health care organization: individual, group/team/community, and organization (Figure 1). This link also constitutes a fruitful way of approaching the complexity of managing $\mathrm{KS} / \mathrm{KT} /$ integrated KT among health care professionals/public health practitioners, and mapping out future directions toward a "transfrontier KT approach" that institutionalized health care KS and KT might take.

\section{"Integrated knowledge translation" in a changing global context: A concurrent mixed-method research design}

Faced with the problem of an ever-mounting growth of information/knowledge, or in some cases "paradoxical information/knowledge", both collective actors (health care institutions) and individual actors (public health practitioners and health scientists) need solutions. At the same time as the information/knowledge overload is increasing, health costs are expanding. Consequently actors at different levels - health care institutions workers, professionals, and researchers - are faced with the unattractive task of discovering approaches that are "better", "faster", and "cheaper". With these imperatives in mind, new concepts, paradigms, or guiding principles reflective of the contemporary forces driving global change have become prominent in the past decade:

i. Evidence-based medicine and the drive for evidence-based approaches 
Table I A note about terminology

Artifact-mediated KT-KS practices involve the application and the sharing of knowledge through an artifact (such as a research paper, clinical practice guidelines) or around an artifact. ${ }^{3}$ In the former case, the artifact (published literature) serves as the knowledge object that can be shared/used through a knowledge database (Medline or Cochrane), or the knowledge can be exchanged through a communication channel (eg, e-mail, Facebook) between stakeholders having a common interest. ${ }^{3}$ In the latter case, the artifact serves as the focal point for a critical discussion between interested stakeholders. In both cases, the artifact serves as the stimulus for KT-KS. The artifact is linked to explicit, concrete knowledge.

Experience-mediated KT-KS practices involve the articulation and application of clinical, operational, and psychosocial experiences, insights about a particular topic. ${ }^{3}$ Health care stakeholders, originating from different backgrounds, engage, collaborate, and share their intrinsic knowledge and extrinsic work experience to address specific issues through a communication medium (eg,Webinar or e-mails exchanges). The types of knowledge involved here: (i) have the relatively limited accessibility of implicit knowledge, and (ii) fall in the categories of contextual and procedural knowledge.

Resource-mediated KT-KS practices involve the identification of knowledge resources as a by-product of actual KT-KS activities between health care stakeholders. ${ }^{3}$ In this case, the associated knowledge resources (eg, domain experts, contact persons, knowledge brokers, websites, discussion forums, knowledge artifacts, etc) are identified, registered, and shared as potential sources of knowledge for a specific health care issue. The types of knowledge involved here are pragmatic knowledge and social knowledge.

Knowledge-sharing: "In the knowledge management parlance, KS can be regarded as a systematically planned and managed activity involving a group of like-minded individuals engaged in sharing their knowledge resources, insights, and experiences for a defined objective". ${ }^{3}$ Heath care KS can be characterized "as the explicitation and dissemination of context-sensitive healthcare knowledge by and for health care [decision-makers] through a collaborative communication medium (...) Health care knowledge-sharing is practiced for a variety of reasons, including clinical decision-making, patient education vis-à-vis patient empowerment programs, practitioner's education and experience enhancement, translation of knowledge to practices and vice versa, health care policy-making, clinical protocol and guideline formulation, public health and community support for patients, capturing care-giver perspectives and feedback about practices and outcomes, and disseminating clinical research findings" (p. 69). ${ }^{3}$ The aim of heath care KS practices can be characterized as:

I) To provide efficient and focused access to evidence-based knowledge "resources" (eg, contact persons, websites, domain experts, discussion forums, knowledge artifacts, etc), either by guiding the user to the knowledge "artifacts" (a research paper, a practice guideline, a document) or by providing peer recommendations to help to find the relevant knowledge artifacts.

2) To share the "unpublished" intrinsic experiential know-how, insights, judgements, and problem-solving strategies of decision-makers to complement evidence-based knowledge.

3) To establish a culture for collaboration between like-minded decision-makers in order to stimulate collaborative learning, atypical problemsolving, practice evaluation, critical appraise of evidence, practices and outcomes, leveraging peer experiences and knowledge. ${ }^{3,4}$

\section{In Canada, the term "KS" is mainly used by health care managers and administrators}

Knowledge translation: The terms "KT" and "KT research" are not synonymous. KT is a human enterprise that can be studied to understand and improve knowledge translation approaches whereas KT research is the scientific study of the determinants, processes and outcomes of knowledge translation. ${ }^{50} \mathrm{KT}$ is a dynamic and iterative process that includes synthesis, dissemination, exchange, and ethically sound application of knowledge to improve the population health, provide more effective health services and products, and strengthen the health care system. ${ }^{76}$

I) "Planning": The initial stage of the KT process, occurring before the doing and evaluation stages. Planning methods and tools are used in the early stages when thinking about how to frame, approach, or organize KT activities. ${ }^{82}$

2) "Doing": The intermediate stage of the KT process that occurs after the planning stage and before the evaluation stage. Doing resources are methods and tools for the active phase of implementation and are used to facilitate or perform knowledge translation activities. ${ }^{82}$

3) "Evaluation": Systematic efforts, using quantitative and/or qualitative approaches, to assess the effectiveness and impact of ongoing or completed KT activities in relation of their objectives. ${ }^{82}$

In Canada, the term "KT" is mainly used by health scientists, clinicians, and public health practitioners

Knowledge translation research: KT research is the scientific study of the determinants, processes, and outcomes of KT. The goal of KT research (KT science) is to develop a generalizable empirical and theoretical basis to optimize KT activities. ${ }^{50} \mathrm{KT}$ research is a new field in health research which is inherently interdisciplinary. A wide range of disciplines need to be engaged (clinical sciences, health services research, health information technology, communications, behavioral and organizational theories, design and engineering, etc) as well as a broad range of forms of enquiry are needed.

Integrated knowledge translation or T2 research: The terms "T2 research" and "integrated KT" are synonymous. The former is mainly used in the USA, ${ }^{54}$ whereas the latter is preferred in Canada. ${ }^{53,36-38}$ Integrated KT or T2 research requires a partnership between the T2 researchers (academic partners) and the practitioners/decision-makers/policymakers/caregivers. The development of this partnership is the hallmark of integrated KT or T2 research.

In Canada, the term "integrated KT" is more and more often used by health scientists and public health practitioners Mode II of knowledge production: Mode II refers to the contextualization of research when research programs combine a social and a scientific relevance. Mode II is based on the assumption that science can no longer be confined to the university; science penetrates the society, while in many ways society influences science..$^{56,68,69}$ Mode II contrasts with Mode I of knowledge production whose substance was science confined within disciplinary boundaries. A Mode II perspective argues for co-operation by researchers for resolving critical problems in a changing global context. The

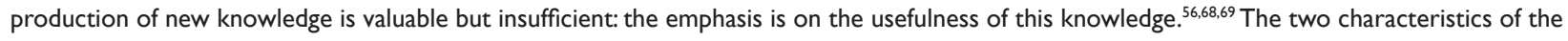
Mode II are: (I) its explicit valuation of research, and (2) intense relationships between researchers and other social actors. In Canada, recent policies by various health research funders are closely aligned with Mode II, and many funding programs foster the development of partnerships in research ("integrated KT" or “T2 research").

(Continued) 
Table I (Continued)

Public health: An organized activity of society to promote, protect, improve, and when necessary, restore the health of individuals, specified groups, or the entire population. It is a combination of sciences, skills, and values that function through collective societal activities and involve programs, services, and institutions aimed at protecting and improving the health of all people. The term "public health" can describe a concept, a social institution, a set of scientific and professional disciplines and technologies, and a form of practice. It is a way of thinking, a set of disciplines, an institution of society, and a manner of practice. It has an increasing number and variety of specialized domains and demands of its practitioners an increasing array of skills and expertise..$^{82}$

Public health practitioner: The term "public health practitioner" includes frontline workers, researchers, professionals, managers, decision-makers and policy-makers.

ii. The drive for a major leadership shift (from a traditionally top-down leadership to a transformational or coalition leadership) in health care

iii. The drive for new models of KS in the knowledge management field, and the emergence of the KT paradigm in health care and public health

iv. The drive for global collaboration and a renewed definition of globalization in public health

v. The drive for acknowledgement of the social nature of many determining factors, and consequently, the drive for new theories, perspectives, and/or identities in public health "[capturing] the idea that the physiological end-pathways leading to an individual's ill health are inextricably linked to the social setting" (p. 14). ${ }^{53}$

vi. The drive for transdisciplinary teams and participatory research (integrated KT) in the health care and public health sectors.

Today's health care organizations increasingly recognize the need to support the practice of KS and KT among their "employees". As a consequence, researchers and professionals in clinical, managerial, and policy decisionmaking environments are collaboratively searching, testing, and using proactive interventions to facilitate $\mathrm{KS}$ and the application of knowledge. However, the success of active and efficient collaborations depends on a myriad of factors.

My two-part research study focused on active collaboration for sustainable KS-KT practices in globally oriented (public) health research. It drew on a nonlinear perspective that conceives $\mathrm{KS}$ and $\mathrm{KT}$ as accomplishing changes not only in technical, but also, and primarily, in cognitive, social and, organizational contexts.

This study was at the crossroads of three specific perspectives of research: (i) KT research, ${ }^{53,56}$ (ii) critical population health research, ${ }^{57-59}$ and (iii) the new field of equity in health. ${ }^{60}$ My research goal was to elaborate an integrated KT conceptual framework designed for public health practitioners and researchers. The methodological design was a concurrent ${ }^{\mathrm{a}}$ mixed method design (QUAN + qual) : (i) the QUAN phase of the QUANqual design was a systematic review method, ${ }^{64}$ and (ii) the qual phase of the QUAN-qual design was a Soft Systems Methodology. ${ }^{65-67}$ The qualitative strand/phase of this methodological approach enables this two-part study to be look upon as an integrated KT, responding to what is been referred as "Mode II of knowledge production" 68,69 (Table 1 and Table 2).

The qual strand was under the umbrella of a larger research program called "PROACTIVE" (Participatory and Evaluative Research Program to Optimize Workplace Management: Application of Knowledge, Transfer of Expertise, Innovative Interventions, Training Transformational Leaders). ${ }^{.}$The participants were health care managers (as "integrated KT users"; $n=199$ [from 34 management teams originating from three Canadian provinces: Quebec, Ontario, and New Brunswick]) and academic partners (as knowledge producers $[\mathrm{n}=13]){ }^{70}$ Data collection included different Web-techniques

\footnotetext{
${ }^{\text {a }} \mathrm{A}$ concurrent mixed method design is a multistrand design in which both qualitative and quantitative data are collected and analyzed to answer a single type of research question (either qualitative or quantitative). The final inferences are based on both data analysis results. The two types of data are collected independently at the same time or with a time lag. ${ }^{61}$

${ }^{b}$ Morse, writing in the applied field of nursing, authored an important paper on approaches to quantitative-qualitative methodological triangulation in which she presented a basic terminology and notational system. ${ }^{62,63}$ Her notational system is the standard currently used in the mixed methods research area. Components of this system include the following: (i) use of the abbreviations QUAN for quantitative and QUAL for qualitative, (ii) use of the plus sign (+) to indicate that data are collected simultaneously (eg, QUAN + qual), and (iii) use of uppercase to denote more priority given to that orientation (eg, QUAN). ${ }^{62,63}$

"PROACTIVE" was a sequential program of three research projects focusing on the reorganization of care, services, and work in Canada. This program was led by Chantal Viens and Sylvie Hains from Laval University and Ministry of Health and Social Services, respectively. ${ }^{70}$ The PROACTIVE program's main objectives were to support Canadian health care institutions' managers in carrying out evidence-based decisions on the reorganization of care, services, and work, to promote transformational leadership, and to mentor these managers and their partners. ${ }^{70}$ Only the secondary results of the PROACTIVE program were considered here.
} 
Table 2 The similarities and differences between KT, KT research, and integrated KT

\begin{tabular}{|c|c|c|}
\hline Knowledge translation & Knowledge translation research & $\begin{array}{l}\text { Integrated knowledge translation } \\
\text { or "T2 research" }\end{array}$ \\
\hline $\begin{array}{l}\text { KT is about: } \\
\text { - Making users aware of knowledge } \\
\text { and facilitating their use of it to improve } \\
\text { health and health care systems; } \\
\text { - Closing the gap between what we } \\
\text { know and what we do (reducing } \\
\text { the know-do gap); } \\
\text { - Moving knowledge into action. } \\
\text { KT is a dynamic process that includes synthesis, } \\
\text { dissemination, exchange and ethically sound } \\
\text { application of knowledge to improve the health } \\
\text { of population, provide more effective health } \\
\text { services and products and strengthen the } \\
\text { health care system. } \\
\text { This process takes place within a complex } \\
\text { system of interactions between researchers } \\
\text { and knowledge users which may vary in } \\
\text { intensity, complexity, and level of engagement } \\
\text { depending on the nature of the research and } \\
\text { the findings as well as the needs of the } \\
\text { particular knowledge user. }\end{array}$ & 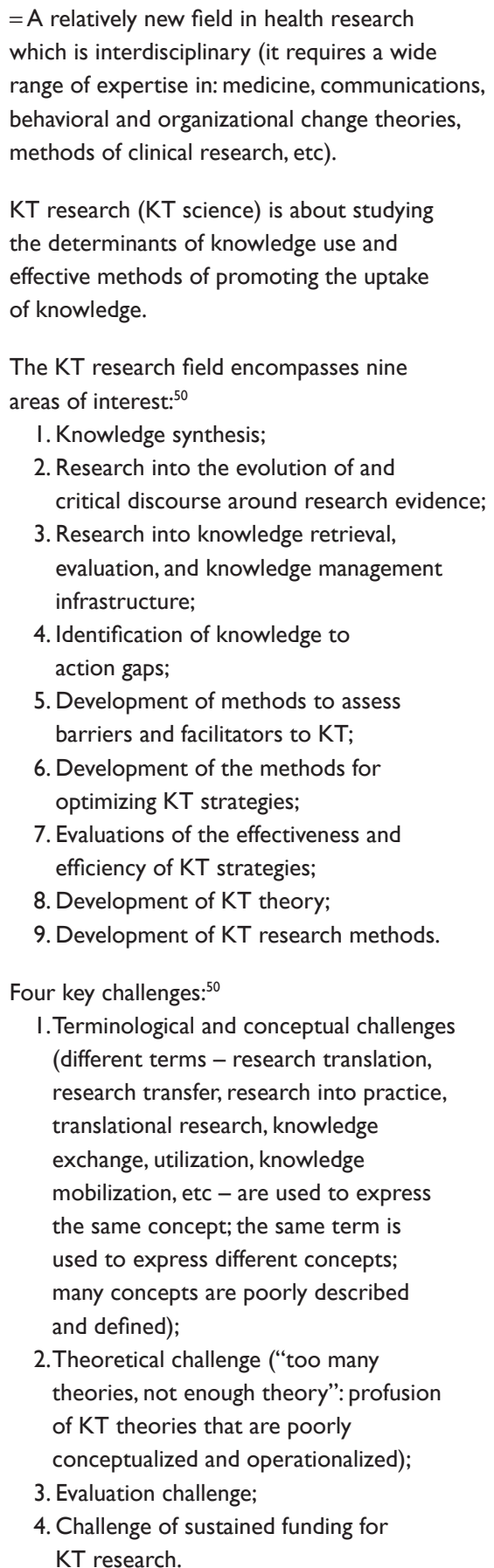 & 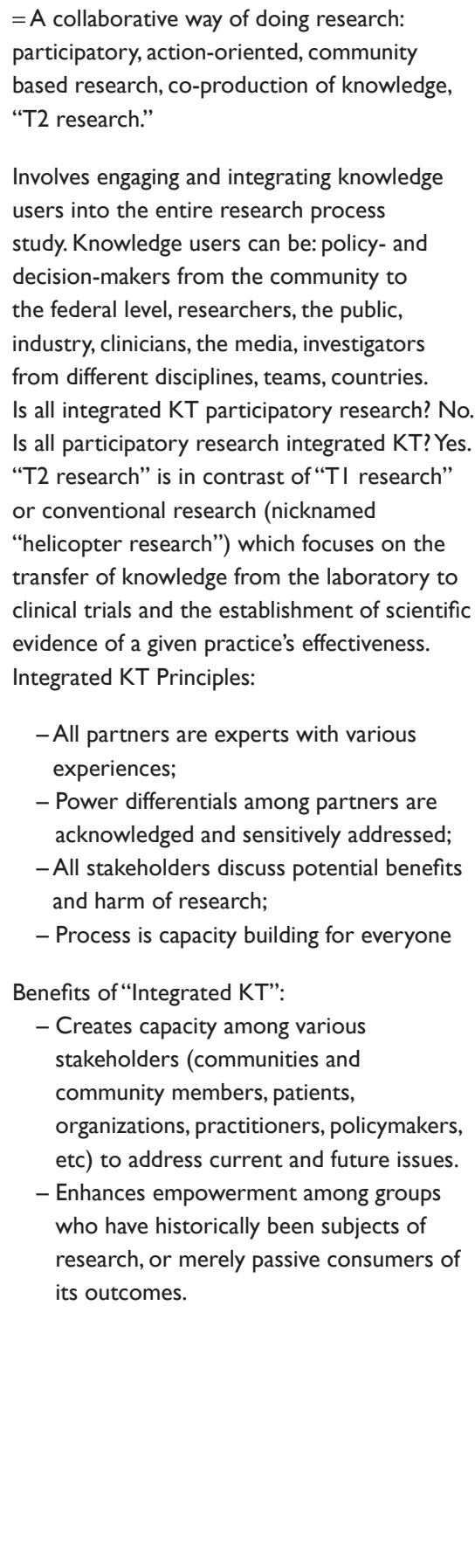 \\
\hline
\end{tabular}

Abbreviation: KT, knowledge translation.

(Web-based journal, Web-based interviews, peer-review Soft Systems Model validation through Web-conferencing), and data analysis was conducted by grounded theory.

The QUAN strand's main objective was to identity the key elements of the dynamic process of integrated KT within health care institutions. The following research questions were considered in this systematic review: (1) What are: (i) the different definitions of "integrated KT" within health care institutions, (ii) the different types of knowledge and forms of evidence used by health care managers/public health practitioners, (iii) the different types of managerial $\mathrm{KS}$, and/or (iv) the determinants 


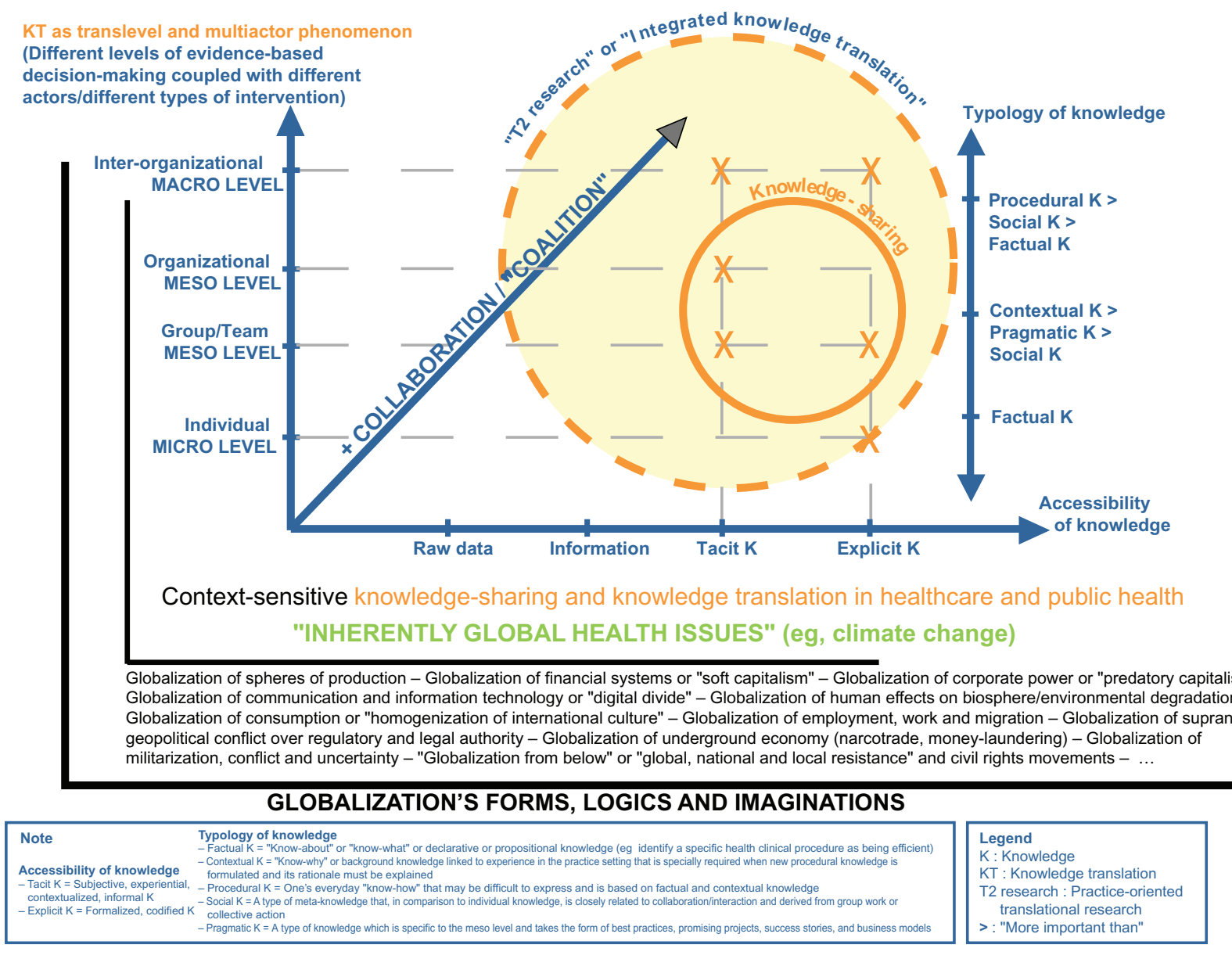

Figure I Integrated knowledge translation in a changing global context.

of this KT?, (2) What are the integrated KT conceptual frameworks discussed in the literature?, and (3) What are the key indicators used to assess the KT evolution within health care institutions?

Two search chains (English and French keywords search chains) were used as follows:

- ("evidence-based health care management" OR "evidencebased healthcare management" OR "evidence-based health management") OR (("knowledge manage*” OR “evidencebased management" OR evidence OR "knowledge transfer" OR "knowledge translation" OR "knowledge sharing" OR "knowledge utilization" OR "research utilization" OR "knowledge application") AND (health OR healthcare OR "health care" OR hospital* OR "health W/2 organization*" OR "health W/2 administrat*" OR "public health W/2 institute*"))

- (((gestion OR administrat* OR cadre* OR organis* OR institut*) W/3 (soins OR service* OR sante)) AND (preuve* OR "donnee* probante*" OR "savoir* probant*" OR “donnee* factuelle*” OR” fait*” OR evidence*)) OR ((gestion OR manage* OR transfert OR utilization OR application) W/3 (savoir* OR connaissance* OR resultat*) AND (soins OR service* OR sante))

The inclusion criteria were: (1) English and French sources, (2) published and unpublished documents or reports, (3) manual searching used as well as electronic databases, and (4) publication period: from 1990 to 2009 . The exclusion criteria were: (1) master or $\mathrm{PhD}$ thesis, (2) editorials, and (3) success stories. The databases consulted were: ISI Web of Knowledge, EBSCO, Cochrane Library, Current Contents: All EBM Reviews: MEDLINE/Pubmed, EMBASE, CABI, CINHAL, ASSIA, AHA Hospital and Health Administration Index, and BDSP. The references were entered into Reference Manager v.11.0 (Thomson ResearchSoft, New York, NY) and after removal of duplicates, a total of 11,207 references remained. These were further reduced through review of abstracts. Results reported here are based on preliminary analysis of 308 articles.

The development of my conceptual framework was based on the secondary results of the qual strand and the preliminary results of the QUAN strand (systematic review). At the end 
of each strand/phase of the design, qualitative inferences (predominantly inductive inferences) were made. These inferences were combined/contracted to achieve a fuller picture of the integrated KT process. ${ }^{71}$ Strategies for integrating these inferences and the role of each (eg, exploratory, triangulation) were more crucial than the priority/dominance of one approach over another (QUAN-qual). ${ }^{72,73}$

\section{What determines "integrated knowledge translation"? A global-context-sensitive conceptual framework}

Integrated KT is influenced by many factors/determinants and groups of factors/determinants that are interrelated at many levels, but can be divided into two supra-categories: (i) "upstream" determinants, and (ii) proximal determinants. Each of these supra-categories can be subdivided in the following way:

I. "Upstream" determinants of integrated KT (context of contemporary globalization)
Ia. Globalization: forms, logics, and imaginations

Ib. Multilevel factors of life-worlds

Ic. Multilevel vulnerability and "inherently global health issues"

II. Proximal determinants of integrated KT

IIa. Health systems characteristics

IIb. Determinants of evidence-based decision-making

IIb-1. Types of knowledge

IIb-2. Typologies of evidence

IIb-3. Determinants of coalition

IIb-4. Determinants of knowledge-sharing

IIc. Traps and questions.

The usefulness of this framework (Figure 2) is firstly to show the importance of multilevel relationships between: (i) contemporary globalization, (ii) the evidence-based decision-making movement, and (iii) the development of effective and sustainable collaboration between knowledge users and producers. ${ }^{16}$ Secondly, Figure 2 illustrates that: (i) integrated $\mathrm{KT}$ is a nonlinear phenomenon with multiple

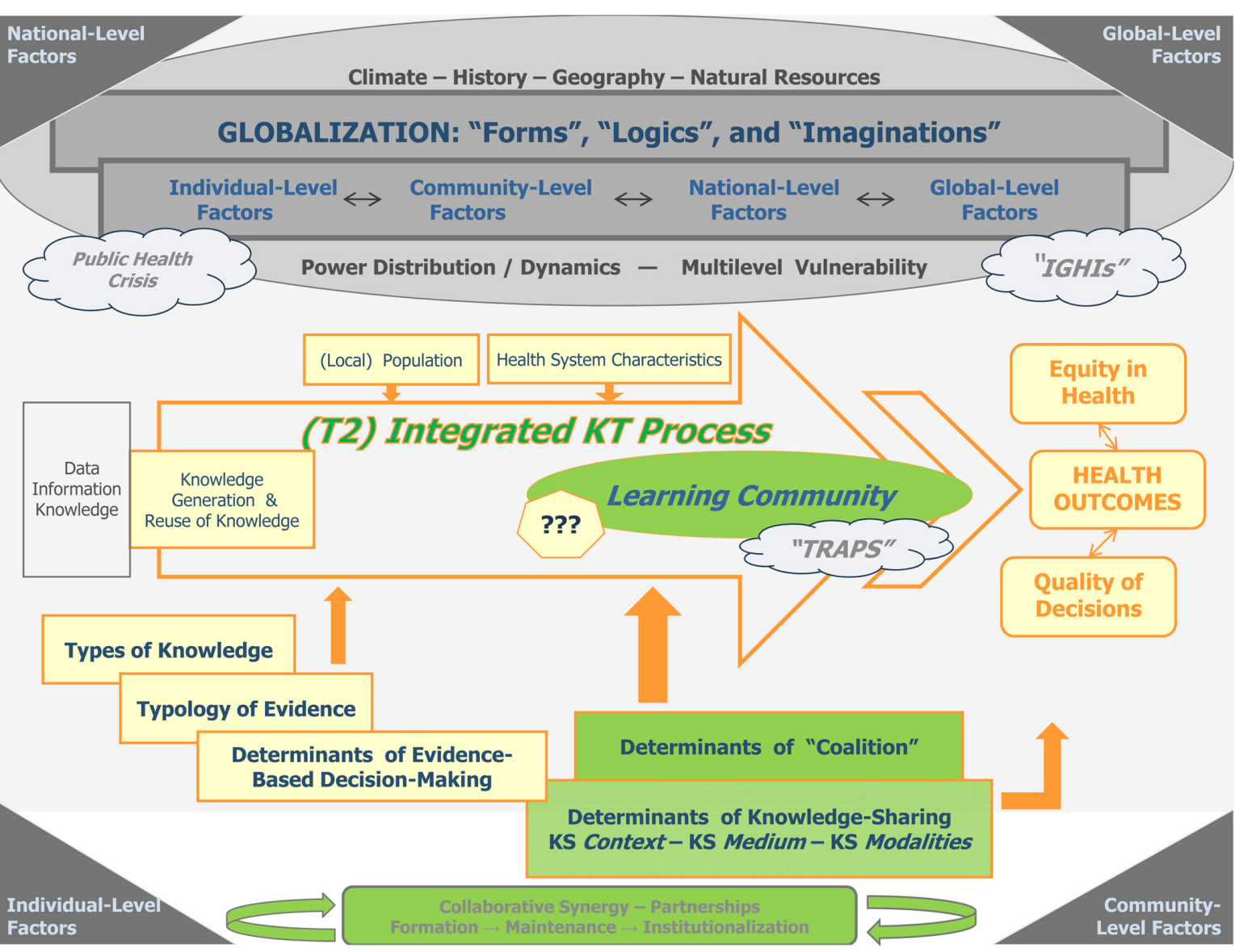

Figure 2 Integrated knowledge translation conceptual framework. 
and complexly intertwined determinants, (ii) integrated KT is a global-context-sensitive phenomenon which is influenced by multilevel factors of life-world and vulnerability (globallevel factors, national-level factors, community-level factors, individual-level factors).

\section{I. “Upstream” determinants of integrated KT (context of contemporary globalization)}

Although globalization (Figure 2) can be described as a sociohistorical process evolving along with human societies, it is necessary to distinguish "globalization" from other terms such as "internationalization" (political internationalization is the growth of cross-border economic and political activity), "liberalization" (economic liberalization refers to "free-market" strategies), "westernization" (the global spread of companies such as McDonald's), and "universalization" (global convergence around cultural and institutional forms), terms that are often used interchangeably. The work of Scholte is helpful in this respect. ${ }^{74}$ This author reserves the term "globalization" to describe social interactions that not only cross national boundaries, but also transcend them, and he sees the emergence of transplanetary relations and the rise of supra-territoriality as genuinely novel features of globalization. ${ }^{74}$ Satellite communications, the Internet, transplanetary $\mathrm{TV}$, intercontinental production chains, anthropogenic ecological changes (eg, global warming), illicit drug trafficking, transworld migrants, and economic remittances are examples of globalization in this strict sense. It is very important to be aware of how the term globalization is defined, for a looser definition suggests that at least some aspects of what people call globalization are not novel to the late 20th century. A strict definition of globalization as the transcendence of territorial boundaries is distinctive of the transition facing human societies worldwide in recent decades.

A second important question is why globalization is occurring. What are the "forms" and "logics" of globalization? And what are some globalization-related conceptual schemes or "imaginations"?

\section{la. Globalization: Forms, logics and imaginations}

The forms (Figure 2 and Figure 1) it takes can be differentiated as follows:

- Globalization of capital or of the finance sector (globalization of financial systems: "soft capitalism")

- Globalization of markets and globalization of spheres of production, commerce, and logistical systems
- Globalization of technology, R\&D, and knowledge

- Globalization of lifestyles, consumerism, and culture (or globalization of consumption, "homogenization" of international culture, cultural challenges)

- Globalization of standards (this particular form has major stakes in global governance, which for its part constitutes an integral part of globalization) and globalization as a worldwide policy instrument (globalization of supranational and geopolitical conflict over regulatory and legal authority)

- Globalization of perceptions of human conditions or of a "global conscience", "globalization from below" (global, national, and local resistance, and human rights movements)

- Globalization of communication and information technology ("digital divide") and communication-media globalization

- Globalization of corporate power or "predatory globalization" (mega-mergers, oligopolies)

- Globalization of human effects on biosphere, and of environmental degradation

- Globalization of militarization, conflict, and "fear and risk" (post-cold War continuity, post-9/11 tensions)

- Globalization of employment, work, and migration.

Furthermore, globalization subscribes to six different "logics", ${ }^{16}$ that can be defined as abstract and schematic orders of reasoning that allows an understanding of the complexity of reality. First, there are market logics and productive logics, which are closely related and make up the core of the new globalizing world. Productive logic is the root of the organizational changes in globalization on which production, re-engineering, and "total quality" are based. Market logic extols the marketability of everything and anything. Secondly, at the very core of the capitalist system is velocicratic logic. In the context of globalization, new forms of expression of the human connection to time are effectively emerging: these are urgency, immediacy, instantaneousness, and speed, with acceleration being the common denominator that unites the other forms. The arrival of urgency and instantaneousness in economic life falls right in line with the emergence of a new global space-time. Velocicratic logic therefore joins another type of logic - cyber logic - which, for its part, is related to the ICT revolution. Technoscientific logic, which represents the alliance between science and technology, comes third. Technoscientific logic is inseparable from the performance culture which, when allied with the cult of speed and subjected to the law of market, gives it real meaning. Coming fourth is epidemic logic, which relates 
to the breakdown of boundaries. Whether we are dealing with a computer virus, the disappearance of borders, the deregulation of the foreign exchange market, a rise in political extremism, or the mad cow crisis, a similar "epidemic" logic seems to guide these apparently social facts.

Moreover, the new globalizing world is shown through various conceptual schemes, or "imaginations":

- The comprehensivist imagination, as in "similar throughout the world"

- The "worldwide everyday life" imagination ("global happenings", catastrophes, etc)

- The "collective cohabitation" imagination

- The "everything is for sale and everything can be bought" imagination, as in freedom of choice

- The discursive imagination (that is, words specific to our times: governance, accountability, effectiveness, quality improvement, and assessment).

These different forms, logics, and imaginations of contemporary globalization influence multiple "levels" of determination of variegated life-worlds. Generally speaking, they cause a profound crisis in public health. More specifically, they can be tied to the development of integrated KT as well as the development of a multilevel vulnerability and the emergence of "inherently global health issues" (IGHIs).

\section{Ib. Multilevel factors of life-worlds}

- Global-level factors include: global trade, global public goods, emerging global governance, ICT, migration, income distribution.

- National-level factors refer to: infrastructural resources, population density, employment, income growth, political institutions, policies and programs which enhance capabilities and build social capital (eg, education, income transfers, public investments in infrastructure, disease prevention measures), social inclusion, and social support networks.

- Community-level factors correspond to: resource allocation, population heterogeneity, social environment, and physical environment (eg, clean water and air, safe and warm housing, safe working conditions, adequate fuel supplies, fertile soil).

- Individual-level factors include: biology and genetics endowment, healthy child development, gender, personal health practices, and coping skills.

\section{Ic. Multilevel vulnerability and "inherently global health issues"}

A new category of vulnerability has appeared in the context of contemporary globalization that can be termed a "globo-agentive" vulnerability, referring to the impact of globalization upon agents at various levels of their life-world. This multilevel vulnerability is itself in interaction with the appearance of IGHIs and the public health crisis (see the upper part of Figure 2).

- Globo-agentive vulnerability is manifested in: income disparities, educational disparities, geospatial disparities (eg, regional inequalities, economic segregation), gender disparities and discrimination, and ethnic disparities.

- Globo-agentive vulnerability results from factors such as: living conditions (eg, housing, neighborhood characteristics), employment and working conditions (economic insecurity, occupational exposures), food quality and security, water quality, and air quality (eg, urban, agricultural and industrial pollution exposures, access to safe water, sanitation).

- Globo-agentive vulnerability is linked to the emergence of IGHIs. Labonte et al proposed the term "inherently global health issues" to describe health-determining phenomena that transcend national borders and political jurisdictions and urge greater research and policy attention to the linkages between these issues. ${ }^{75}$ IGHIs are related to the crisis of public health; they can be structured around three themes: ${ }^{76}$

- The first theme concerns collective resources and comprises climate change, the disappearance of ecosystems, the diminution of marine resources, deforestation, water shortage, and maritime pollution.

- The second theme involves action by individual citizens, with respect to the intensification of the struggle against poverty, the struggle against terrorism, education for every person, infectious disease, the digital divide, and the prevention and management of natural catastrophes.

- The third theme relates to burgeoning global governance. With respect to codes, norms, and regulations, it will be necessary to: (i) re-invent income tax for the 21 st century, (ii) reflect on the establishment of rules concerning biotechnology, a worldwide financial structure, commerce, investment, and competition, (iii) reflect on laws concerning intellectual property, and (iv) reflect on the establishment of rules of commerce, as well as international rules concerning work and migration.

\section{Proximal determinants of integrated knowledge translation}

The components of an integrated KT process include both health care characteristics and the determinants of 
evidence-based decision making which can be characterized as (i) types of knowledge, (ii) evidence typology, (iii) coalition determinants, and (iv) KS determinants (see the lower part of Figure 2). Collectively, in a nonlinear fashion, all of these factors decisively impact the integrated KT process, which in turn influences health outcomes, decision quality, and health equity. Finally, in the case of an integrated KT process involving the development of a learning community, there are traps to be avoided and questions that must be asked to assure the success of the integrated KT.

\section{Ila. Health system characteristics}

These characteristics include: the availability of care, the affordability of care (eg, effects of cost recovery, user charges), and the availability of care (eg, adequate health human resources, rural/urban equity).

\section{Ilb. Determinants of evidence-based decision-making Ilb-I.Types of knowledge}

Five types of knowledge can be distinguished in the health and public health sectors: (i) factual knowledge (propositional knowledge or "Know-About"), (ii) contextual knowledge ("Know-Why"), (iii) procedural knowledge ("Know-How"), (iv) social knowledge, and (v) pragmatic knowledge. ${ }^{77}$

Types of knowledge can further be distinguished with respect to relative accessibility: (i) easily-accessible explicit knowledge (formalized, codified knowledge), (ii) less-accessible tacit knowledge (subjective, experiential, contextualized, informal knowledge), and (iii) implicit knowledge considered as having "intermediate" accessibility.

\section{Ilb-2. Typologies of evidence}

With respect to the various types of evidence, the following three aspects should be considered:

- The sources of evidence: published literature and grey literature; "absolute scientific data", "contextual scientific data", and "informal data"

- The nature of evidence: qualitative research, survey, casecontrol studies, cohort studies, randomized control trials, quasiexperimental studies, nonexperimental interventions, and systematic reviews

- The characteristics of scientific evidence: availability, accessibility, validity, timing, and communicability.

\section{Ilb-3. Determinants of coalition}

Within an integrated KT process, the determinants of coalition have to be taken into account. Empowering people to develop a collective vision and creating systems to capture and share learning (in case of the development of a learning community) is not an easy undertaking. The determinants of coalition are: the structural and functional characteristics of coalition such as formalization, communication, conflict resolution, as well as the commitment to collaborative efforts, the perceived benefits, and the institutionalization of the community.

\section{Ilb-4. Determinants of knowledge-sharing}

The determinants of KS include: (i) the KS context, (ii) the KS medium, and (iii) the KS modalities.

- KS takes place within a context which epitomizes: (i) the issue or the subject of the shared knowledge, (ii) the motivation for KS, (iii) the temporal relevance of the shared knowledge, (iv) the orientation of the decision-makers engaged in the KS exercise (eg, terminology used, brevity and formality of knowledge, language constructs, expected outcomes, trust in sources), and (v) the decision-makers' needs, interests, operational capacity, and degree of trust towards the knowledge.

- $\mathrm{KS}$ is practiced via a medium. The sophistication of this medium may range from face-to-face environments (eg, person-person conversations, speaker-audience interactions) to Internet-based mediums (eg, online discussion forum, Web-CT, peer-to-peer networks).

- KS modalities are determined by the nature of the knowledge to be shared (explicit versus implicit, factual versus contextual, pragmatic versus social) as well as the character of the relevant evidence (availability, accessibility, validity, timing, and communicability). The KS modalities can be characterized in the following ways: (i) asynchronous versus synchronous versus multisynchronous, (ii) anonymous versus disclosed identity of the decision-makers, (iii) individual-based interactions versus group interactions, (iv) push versus pull versus deliberative mode of knowledge delivery, (v) open participation versus membersonly participation, (vi) supporting-dynamic versus static knowledge content, and (vii) short-term versus long-term relationships between decision-makers.

\section{Ilc. Traps and questions}

In the case of an integrated KT associated with the constitution of a learning community, the integrated KT process can be analyzed by answering to the following questions:

- Why is knowledge shared?

- When is knowledge shared?

- Where is knowledge shared?

- Who shares knowledge? 
- What type of knowledge is shared?

- How is knowledge shared? (these questions are represented with question marks in the center of Figure 2).

One must also be watchful for the various "traps" that can complicate the successful development of a learning community's integrated KT:

- The "individual learning trap" (this trap refers to the danger of treating integrated KT as a learning process for the individual [at the micro level] while disregarding its contribution to the health care institution/health sector [at the meso/macro level])

- The "ICT trap" (this trap revolves around placing too much faith in information and communication technologies' ability to improve integrated KT)

- The "management trap" (this trap relates to the tendency to approach the need for supporting integrated KT with too much empathy for the management perspective)

- The "inequity trap" (this trap related to the tendency to forget the context of contemporary globalization and IGHIs).

\section{Conclusion: The need for a transfrontier approach in knowledge translation research}

Success-determining factors of an integrated KT with respect to a given IGHI are numerous, varied, and interrelated in complex ways: their effective application is no easy task. In Quebec, a recently-developed approach that takes into account a maximum number of these factors is being applied to the specific IGHI of climate change. The KT research chair, Climat, santé, écoapprentissages, provides an illustration of an equitable, sustainable, and transborder vision in $\mathrm{KT}$ research. ${ }^{78}$

In the face of the planetary challenge of global warming, all of us must act, as citizens and public health workers, if we hope to see humanity develop in a harmonious, just, and environmentally-friendly way. As public health professionals, we can and must do more than simply echo a general appeal for global ecocitizenship: we can promote, develop, and transfer ecoknowledge conducive to healthy climatic conditions: (1) in prioritizing research on the applications of ecoknowledge (KT research), and (2) by orienting this research so that it focuses on the adaptation of public health to climate change, and on the attenuation of climate change (mitigation).

The research program of this Chair respects the global priorities mentioned in Article 6 of the Climate Convention
(United Nations Framework Convention on Climate Change), and the Plan d'action 2006-2012 sur les changements climatiques -Volet santé of the Ministry of Health and Social Services of Quebec. ${ }^{79,80}$ The recent accumulation of research results flowing from studies on climate change poses the challenge of applying these results to achieve attenuation of climate change as well as adapting public health to the impacts of those climate changes.

The Chair promotes values of social justice, enduring change, and shared responsibility, and the explicit intention is to extend application of research results beyond several frontiers (sectorial, disciplinary, geographic, cognitive, and cultural), and is thus in many senses of the term "transfrontier". The Chair is also committed to an overarching vision of: (1) an engaged citizenry with leadership shared among individual and organizational members, and (2) a transfrontier application of knowledge that seeks to reinforce the adaptive capacities of health systems, enabling them to promote climatic health that is just, enduring, "transfrontier", and that entails shared responsibility. The "transfrontier" aspect of this vision $^{78}$ refers to an application of knowledge that would ideally be:

i. Trans-sectorial, in that the program seeks to transcend hermetic sectorial compartmentalization of knowledge among key actors for adaptation

ii. Transdisciplinary, in that the program seeks to break down disciplinary barriers (climate sciences, public health sciences, social sciences) and exclusionary schools of thought between key actors

iii. Transnational (and transinstitutional), in that the program seeks to transcend spatial boundaries between agents as well as spatiotemporal boundaries between their knowledge bases

iv. Transcognitive, in that the program seeks to transcend the cognitive forms that characterize various disciplines, that may facilitate work and communication within a discipline but render it less accessible to other specialists, as well as to transcend the various supports, medias, and practices related to these disciplines

v. Transknowledge level, in that the program seeks to integrate the various levels of knowledge (micro, meso, macro)

vi. Transcultural, in that the program seeks to transcend cultural and ethnic boundaries, by promoting local cultures of contributing agents (eg, traditional knowledge and practices), mobilizing and integrating them without excluding their particularities. 
In conclusion, this transfrontier KT vision is aligned with Wallace's definition of globalization as "[...] an awareness of how "what affects one affects all", or a consciousness of our fundamental interdependence as a global community, as well as the resulting process of learning to work collaboratively and share and disperse resources within our global community to ensure social justice, equity, the protection of the human rights, and the sustainability of the planet" (p. 19). ${ }^{81}$

Such a consciousness and KT approach can also guide the weeding out of the destructive forms, logics, and imaginations of contemporary globalization. Transfrontier collaboration is needed to fully actualize that which is constructive. The drive for globalization, as newly defined, and transfrontier collaboration research are the pillars of the newly emerging KT research field.

\section{Acknowledgments}

Funding has been provided by the Canadian Health Services Research Foundation (CHSRF) and the Canadian Institutes of Health Research (CIHR) (\# PDA-1833-05). The views expressed in this paper are those of the author and do not necessarily reflect the position or policy of the Canadian Health Services Research Foundation, the Canadian Institutes of Health Research, or the Laval University. This paper was presented during the 137th APHA (American Public Health Association) Annual Meeting which took place in Philadelphia, PA from November 7-11, 2009. It received positive feedback from Dr. George Washington, the facilitator of Session 4036.0 "Translation of health administration theory and research to health administration practice". The author equally thanks Dr. Nancy Borkowski, Director of the Chapman Graduate School of Business, from Florida International University, as well as Dr. Lea Ayers LaFave of the New Hampshire Public Health Institute.

When the study was conceptualized and designed, Veronique Lapaige was a CHSRF/CIHR knowledge translation research fellow in the PROACTIVE program (Participatory and Evaluative Research Program to Optimize Workplace Management: Application of Knowledge, Transfer of Expertise, Innovative Intervention, Training Transformational Leaders), a research program directed by Chantal Viens and Sylvie Hains. At the time of the study, the author was mentored by Dr. Chantal Viens (Laval University), Dr. Michel O’Neill (Laval University), Dr. Jean Rochon (Quebec National Institute of Public Health), and Sylvie Hains (Quebec Ministry of Health and Social Services). The author thanks all of them for their thoughtful feedback on the conception of this study.

The author extends her sincere thanks to Christian Dagenais (Montreal University) and his KT research team ("Valorisation et transfert de connaissances" Team) as well as to Marie Désilet and the Louis-H Lafontaine Hospital's librarians (Montreal) for their unconditional support during the systematic review.

Her thanks also go to the reviewers for their insights, helpful comments and precious suggestions.

\section{Disclosure}

The author reports no conflicts of interest in this work.

\section{References}

1. Foray D. Optimizing the use of knowledge. In: Kahin B, Foray D, editors. Advancing Knowledge and the Knowledge Economy. Boston, MA: MIT Press; 2006:9-18.

2. Dolfsma W. Knowledge Economies: Organization, Location and Innovation. New York, NY: Routledge; 2008.

3. Abidi SSR. Healthcare knowledge sharing: purpose, practices, and prospects. In: Bali RK, Dwivedi AN, editors. Healthcare Knowledge Management. Issues, Advances, and Successes. New York, NY: Springer; 2007;67-86.

4. Bali RK, Dwivedi AN, editors. Healthcare Knowledge Management. Issues, Advances, and Successes. New York: Springer; 2007.

5. Wickramasinghe N, Gupta JND, Sharma S. Creating Knowledge-Based Healthcare Organizations. London and Hershey, PA: Idea Group Publishing; 2005.

6. Cowell R. Knowledge management essential, not optional. Nurs Manage. 2006;13(6): 10-13.

7. Chunharas S. An interactive integrative approach to translating knowledge and building a "learning organization" in health services management. Bull World Health Organ. 2006;84(8): 652-657.

8. Walshe K, Rundall TG. Evidence-based management: from theory to practice in health care. Milbank Q. 2001;79(3):429-457.

9. Gray M. Evidence-based Healthcare and Public Health. How to make decisions about health services and public health. 3rd ed. Edinburgh, UK: Churchill Livingstone Elsevier; 2009.

10. Armstrong R, Waters E, Roberts H, Sandy O, Popay J. The role and theoretical evolution of knowledge translation and exchange in public health. J Public Health. 2006;28(4):384-389.

11. Davis D. Continuing education, guideline implementation, and the emerging transdisciplinary field of knowledge translation. J Contin Educ Health Prof. 2006;26(1):5-12.

12. Graham ID, Tetroe J. Whither knowledge translation: an international research agenda [comment]. Nurs Res. 2007;56(4 Suppl):S86-S88.

13. Hedges JR. The knowledge translation paradigm: historical, philosophical, and practice perspectives. Acad Emerg Med. 2007;14(11):924-927.

14. Williams CA. Preparing the next generation of scientists in translation research. Worldviews Evid Based Nurs. 2004;1 Suppl 1:S73-S77.

15. Ramaswami A, Zimmerman JB, et al. Integrating developed and developing world knowledge into global discussions and strategies for sustainability. 2. Economics and governance. Environ Sc Technol. 2007;41(10):3422-30.

16. Lapaige V. La Santé Publique Globalisée [Globalized Public Health]. Québec, Canada: Les Presses de l’Université Laval; 2009.

17. Pablos-Mendez A, Shademani R. Knowledge translation in global health. J Contin Educ Health Prof. 2006;26(1):81-86.

18. Zwarenstein M, Reeves S. Knowledge translation and interprofessional collaboration: where the rubber of evidence-based care hits the road of teamwork. J Contin Educ Health Prof. 2006;26(1):46-54.

19. Weinstein SM. Strategic partnerships: bridging the collaboration gap. J Infus Nurs. 2004;27(5):297-301.

20. Israel BA, Parker EA, et al. Community-based participatory research: lessons learned from the Centers for Children's Environmental Health and Disease Prevention Research. Environ Health Perspect. 2005;113(10):1463-1471. 
21. Israel BA, SchulzAJ, Parker EA, Becker AB. Review of community-based research: assessing partnership approaches to improve public health. Annu Rev Public Health. 1998;19:173-202.

22. Canadian Health Services Research Foundation. Issues in Linkage and Exchange Between Researchers and Decision makers. Available from: http://www.chsrf.ca/knowledge_transfer/pdf/linkage_e.pdf. Accessed September 14, 2008.

23. Jones L, Wells K. Strategies for academic and clinician engagement in community-participatory partnered research. JAMA. 2007;297(4): 407-410.

24. Macaulay AC. Promoting participatory research by family physicians. Ann Fam Med. 2007;5(6):557-560.

25. Minkler M, Wallerstein N, editors. Community-Based Participatory Research for Health: From process to outcome. 2nd ed. San Francisco, CA: Jossey-Bass, 2008.

26. Lapaige V, Gosselin P, Bélanger D. Santé et Adaptation aux Changements Climatiques: Quel transfert de connaissances intégré et comment? [Colloque organisé en partenariat avec l'Institut national de santé publique du Québec]. 78 ${ }^{\mathrm{e}}$ Congrès de 1'Association francophone pour le savoir (ACFAS); 2010 May 10; Montreal, Canada.

27. Baumbusch J, Semeniuk P, McDonald H, et al. Easing the transition between hospital and home: translating knowledge into action. Can Nurse. 2007;103(8):24-29.

28. Boissel JP, Amsallem E, Cucherat M, Nony P, Haugh MC. Bridging the gap between therapeutic research results and physician prescribing decisions: knowledge transfer, a prerequisite to knowledge translation. Eur J Clin Pharmacol. 2004;60(9):609-616.

29. Bowen S, Martens P, The Need to Know Team. Demystifying knowledge translation: learning from the community. J Health Serv Res Policy. 2005;10(4):203-211.

30. Bucknall T. Knowledge transfer and utilization: implications for home healthcare pain management. J Healthc Qual. 2006;28(1):12-19.

31. Curtis LJ, MacMinn WJ. Health-Care Utilization in Canada: 2 Years of Evidence. SEDAP Research Paper No. 190, McMaster University, Ontario. 2007.

32. Drake RE, Goldman HH, Leff HS, et al. Implementing evidence-based practices in routine mental health service settings [comment]. Psychiatr Serv. 2001;52(2):179-182.

33. Estabrooks CA, Thompson DS, Lovely JJE, Hofmeyer A. A guide to knowledge translation theory. J Contin Educ Health Prof. 2006;26(1):25-36.

34. Frank J, Di Ruggiero E, Mowat D, Medlar B. Developing knowledge translation capacity in public health: the role of the National Collaborating Centers. Can J Public Health. 2007;98(4):I1-12.

35. Gagnon MP, Legaré F, Fortin JP, Lamothe L, Labrecque M, Duplantie J. An integrated strategy of knowledge application for optimal e-health implementation: a multi-method study protocol. BMC Med Inf Decis Mak. 2008;8:17.

36. Graham ID, Logan J. Innovations in knowledge transfer and continuity of care. Can J Nurs Res. 2004;36(2):89-103.

37. Graham ID, Logan J, Harrison MB, et al. Lost in knowledge translation: time for a map? J Contin Educ Health Prof. 2006;26(1):13-24.

38. Grimshaw JM, Santesso N, Cumpston M, et al. Knowledge for knowledge translation: the role of the Cochrane Collaboration. J Contin Educ Health Prof. 2006;26(1):55-62.

39. Hunink M, Glasziou P. Decision Making in Health and Medicine. Integrating Evidence and Values. Cambridge, MA: Cambridge University Press; 2005.

40. Kerner JF. Knowledge translation versus knowledge integration: a "funder's" perspective. J Contin Educ Health Prof. 2006;26(1):72-80.

41. Kilian BJ, Binder LS, Marsden J, et al. The emergency physician and knowledge transfer: continuing medical education, continuing professional development, and self-improvement. Acad Emerg Med. 2007;14(11):1003-1007.

42. Landry R, Amara N, Pablos-Mendez A, Shademani R, Gold I. The knowledge-value chain: a conceptual framework for knowledge translation in health. Bull World Health Organ. 2006;84(8):597-602.
43. Lavis JN. Research, public policymaking, and knowledge-translation processes: Canadian efforts to build bridges. J Contin Educ Health Prof. 2006;26(1):37-45.

44. Mitton C, Adair CE, McKenzie E, Patten SB, Waye Perry B. Knowledge transfer and exchange: review and synthesis of the literature. Milbank $Q$. 2007;85(4):729-768.

45. Rycroft-Malone J. Theory and knowledge translation: setting some coordinates [comment]. Nurs Res. 2007;56(4 Suppl):S78-S85.

46. Shortell SM, Rundall TG, Hsu J. Improving patient care by linking evidence-based medicine and evidence-based management. Jama. 2007;298(6):673-676.

47. Straus SE, Graham ID, Mazmanian PE. Knowledge translation: resolving the confusion. J Contin Educ Health Prof. 2006;26(1):3-4.

48. Thompson GN, Estabrooks CA, Degner LF. Clarifying the concepts in knowledge transfer: a literature review. J Adv Nurs. 2006;53(6): 691-701.

49. Titler MG. Methods in translation science. Worldviews Evid Based Nurs. 2004;1(1):38-48

50. Grimshaw J. KT research. CIHR IHSPR-IPPH 7th Annual Summer Institute: Innovation in knowledge translation research and knowledge translation; 2008 June 22-25; Cornwall, Ontario, Canada.

51. Beck U. Risk Society: Towards a new modernity. London, UK: Sage Publications; 1992.

52. Foray D. The Economics of Knowledge. Boston, MA: MIT Press; 2004.

53. Canadian Institutes of Health Research. About Knowledge Translation. Ottawa, Canada: CIHR. Available from: http://www.cihr-irsc.gc.ca/ e/29418.html. Accessed September 17, 2009.

54. Agency for Healthcare Research and Quality. Translating Research into Practice. Available from: http:/grandts.nih.gov/grants/guide/pafiles/PA-02-066.html. Accessed October 10, 2006.

55. Diderichsen F, Evans T, Whitehead M. The social basis of disparities in health. In: Evans T, Whitehead M, Diderichsen F, Bhuiya A, Wirth M, editors. Challenging Inequities in Health: From Ethics to Action. New York, NY: Oxford University Press, 2001.

56. Denis JL, Lehoux P, Champagne F. A knowledge utilization perspective on fine-tuning dissemination and contextualizing knowledge. In: Lemieux-Charles L, Champagne F, editors. Using Knowledge and Evidence in Health Care. Multidisciplinary Perspectives. Toronto, Canada: University of Toronto Press; 2004:18-40.

57. Labonté R, Schrecker T, Packer C, Runnels V, editors. Globalization and Health-Pathways, Evidence and Policy. New York, NY: Routledge; 2009.

58. Lee K. Health Impacts of Globalization: Towards Global Governance. Basingstoke, UK: Palgrave Macmillan; 2003.

59. Lee K. Globalization and Health: An Introduction. Basingstoke, UK: Palgrave Macmillan; 2004.

60. Wallace BC, ed. Toward Equity in Health: A new global approach to health disparities. New York, NY: Springer; 2008.

61. Tashakkori A, Teddlie C, editors. Handbook of Mixed Methods in Social and Behavioral Research. Thousand Oaks, CA: Sage Publications; 2003.

62. Morse JM. Approaches to qualitative-quantitative methodological triangulation. Nurs Res. 1991;40(2):120-123.

63. Morse JM. Principles of mixed methods and multimethod research design. In: Tashakkori A, Teddlie C, editors. Handbook of Mixed Methods in Social and Behavioral Research. Thousand Oaks, CA: Sage Publications; 2003:189-208.

64. Lavis JN, Davies H, Oxman A, Denis JL, Golden-Biddle K, Ferlie E. Towards systematic review that inform health care management and policy making. Res Pol. 2005;10(Suppl 1):35-48.

65. Checkland PB, Scholes J. Soft Systems Methodology in Action. Chichester, UK: John Wiley and Son; 1990.

66. Fennessy G, Burstein F. Using Soft Systems as a Methodology for Researching Knowledge Management Problems. Monash, Australia: School of Information Management and Systems, Monash University; 2006. Available from: http://ftp.informatik.rwth-aachen.de/Publications/CEUR-WS/ Vol-72/026\%20Fennessy\%20SSM.pdf. Accessed September 14, 2008. 
67. Fennessy G. Developing a Model for Knowledge Management within the Context of Evidence-Based Health Care. SHIMR 2000 5th International Symposium on Health Information Management Research; 2000 June 12-13; Sheffield, UK.

68. Gibbons M, Limoges C, Nowotny H, Schwartzman S, Scott P, Trow M, editors. Introduction. In: Gibbons M, Limoges C, Nowotny H, Schwartzman S, Scott P, Trow M, editors. The New Production of Knowledge: The Dynamics of Science and Research in Contemporary Societies. London, UK: Sage Publications; 1994:1-16.

69. Nowotny H, Scott P, Gibbons M. Re-thinking Science: Knowledge and the Public in an Age of Uncertainty. Cambridge: Polity Press; 2001.

70. Viens C, Hains S, et al. Programme de Recherche participative et évaluative visant l'Optimisation de la gestion des milieux de travail. Application de Connaissances, Transfert d'expertise, Interventions novatrices, Formation de leaders transformationnels (Pro-Actif). Canadian Health Services Research Foundation (FCRSS). 2006-2010.

71. Miller S. Impact of mixed methods and design on inference quality. In: Tashakkori A, Teddlie C, editors. Handbook of Mixed Methods in Social and Behavioral Research. Thousand Oaks, CA: Sage Publications; 2003:423-455.

72. Erzberger C, Kelle U. Making inferences in mixed methods: the rules of integration. In: Tashakkori A, Teddlie C, editors. Handbook of Mixed Methods in Social and Behavioral Research. Thousand Oaks, CA: Sage; 2003:457-488.

73. Erzberger C, Prein G. Triangulation: validity and empirically based hypothesis construction. Qual Quant. 1997;2:141-154.

74. Scholte JA. Globalization - A Critical Introduction. 2nd ed. Basingstoke, UK: Palgrave Macmillan; 2005.
75. Labonte R, Spiegel J. Setting global health research priorities. BMJ 2003;326:722-723.

76. Rischard JF. High Noon. New York, NY: Basis Books; 2002.

77. Lapaige V. Evidence-based decision-making within the context of globalization: a "why-what-how" for leaders and managers of health care organizations. Risk Man Healthc Pol. 2009;2:35-46.

78. Lapaige V. Projet de programme de chaire. Chaire d'application transfrontières des savoirs "Climat, santé, écoapprentissages". En collaboration avec 1'Institut national de santé publique du Québec (P. Gosselin). Présenté au Comité directeur du Plan d'action 20062012 sur les changements climatiques-Volet santé. Québec, Canada; 2009.

79. Gouvernement du Québec, Ministère du Développement durable, de l'Énergie et des Parcs. Le Québec et les changements climatiques. Un défi pour l'avenir. Québec, Canada: Gouvernement du Québec; 2008.

80. Gouvernement du Québec, Ministère du Développement durable, de l'Énergie et des Parcs. Troisième bilan de la mise en ceuvre du plan d'action 2006-2012 sur les changements climatiques. Le Québec en action. Québec, Canada: Gouvernement du Québec; 2009.

81. Wallace BC. Introduction: the forces driving and embodied within the new field of equity in health. In: Wallace BC, ed. Toward Equity in Health: A New Global Approach to health Disparities. New York, NY Springer; 2008:1-38.

82. Registry of Knowledge Translation. Glossary. Hamilton, Ontario: National Collaborating Centre for Methods and Tools. Available from: http://www. nccmt.ca/registry/glossary-eng.html. Accessed May 26, 2009.
Journal of Multidisciplinary Healthcare

\section{Publish your work in this journal}

The Journal of Multidisciplinary Healthcare is an international, peerreviewed open-access journal that aims to represent and publish research in healthcare areas delivered by practitioners of different disciplines. This includes studies and reviews conducted by multidisciplinary teams as well as research which evaluates the results or conduct of such teams or

\section{Dovepress}

healthcare processes in general. The journal covers a wide range of areas and welcomes submission from practitioners at all levels, from all over the world. The manuscript management system is completely online and includes a very quick and fair peer-review system. Visit http://www.dovepress.com/testimonials.php to read real quotes from published authors. 